\section{Does the Earth really move?}

SIR-John Durant (Britain's first Professor of the Public Understanding of Science) et al. (Nature 340, 11-14; 1989) complain that "only 34 per cent of Britons and 45 per cent of Americans ... were able to state correctly both that the Earth goes round the Sun and that it takes a year to do so". They clearly implied that the 30 per cent of Britons who responded that the Sun goes round the Earth are mistaken, and they actually stated that "most of the public appear not to have caught up with Nicholas Copernicus and Galileo Galilei". But look what Albert Einstein and Leopold Infeld wrote in their book The Evolution of Physics (Cambridge University Press, 1938, p. 224):

Can we formulate physical laws so that they are valid for all coordinate systems (cs), not only those moving uniformly, but also those moving quite arbitrarily, relative to each other? If this can be done, our difficulties will be over. We shall then be able to apply the laws of nature to any cs. The struggle, so violent in the early days of science, between the views of Ptolemy and Copernicus would then be quite meaningless. Either cs could be used with equal justification. The two sentences, "the sun is at rest and the earth moves", or "the sun moves and the earth is at rest", would simply mean two different conventions concerning two different cs. Could we build a real relativistic physics valid in all cs; a physics in which there would be no place for absolute, but only for relative motion? This is indeed possible!

This important lengthy passage is quoted in full lest the incredulous reader might not trust this writer's word. Most of the public may not have caught up with Copernicus and Galilei, but it appears that most of the professors have not caught up with Einstein. Should not the public expect professors of the public understanding of science to be acquainted with the most basic facts of science before they complain that most of the public are ignorant of scientific matters?

THEO THEOCHARIS

Department of Physics,

Imperial College,

Blackett Laboratory,

Prince Consort Road,

London SW7 2BZ, UK

\section{Reasonable doubt}

SIR-Eric S. Lander's Commentary and your accompanying leading article (Nature 339, 491 and 501; 1989) raise the question of reconciling scientific and legal thinking. However, while the mere fact of the appearance of these publications demonstrates that science is pursuing its usual practice of self-criticism, I have seen no evidence that the law is doing anything to modify its practices in the light of scientific advance.

I wish to raise here two issues involving probability, which figures in both the contributions referred to. While most would agree that someone cannot be " 60 per cent guilty", it is quite possible that there may be an $x$ per cent probability of guilt. In some cases the probability can be quantified with some exactness, and the question then arises - what probability represents the 'reasonable doubt' beloved of the legal profession?

In my experience, lawyers do not want to address this question, perhaps because it takes the matter out of their hands and puts it in the hands of scientists. But surely a legal consensus should exist, and be known to all members of the profession.

A second issue (which crops up quite frequently in the courts) is where death rates due to a given illness in a particular cohort are raised above the normal level by local external circumstances. When the arguments are purely statistical, we may know that $n+m$ individuals have died instead of the expected $n$, but it is impossible to determine whether any particular individual is one of the $m$ or one of the $n$. In other words, it is impossible to say in any individual case whether the external circumstances were or were not the cause of death. I have never heard of this argument being raised in any of the large number of cases involving this type of circumstances that have been or are being tried.

It will be interesting to see whether lawyers are prepared to exercise the same sort of self-criticism as do scientists, and review their attitudes to circumstances such as those described above (there are many others) where science is at present either ignored or distorted.

GEORGE F. WOOD

Parkfield, Charlton, Tetbury,

Gloucestershire GL8 8TS, UK

\section{Complications}

SIR-In a News and Views article (Nature 338, 457; 1989), John Maddox uses the word "complicated", but I think "complex" is the right word. The word 'complex' is now a technical term used by physicists and computer scientists. A non-living static structure can be very complicated, but it need not be complex. Complexity results from dynamics. If a structure can, in principle, be described completely, it is not complex. Complicated non-living static structures are not complex because they can be described with sufficient accuracy in a finite time.

Complexity of dynamic systems is connected with unpredictability. In classical mechanics, it is assumed that the entire future is predictable in a deterministic system. But this is true only for linear deterministic systems.
Linear systems are those that can be divided into parts, and each part can be studied in isolation. Not much information is lost when the system is divided. The Solar System can be linearized in the sense that Earth-Sun, Earth-Moon, Sun-Jupiter subsystems of the Solar System can be studied in isolation without significant loss in the accuracy of the results. That the eclipses can be predicted so accurately exemplifies that linear systems are not complex.

Nonlinear deterministic systems, on the other hand, are complex. "Deterministic chaos" (Gleick, J. Chaos, Heinemann, London, 1988) is an example of a complex system. A chaotic system can be modelled with simplistic deterministic equations, yet the model cannot be used to predict the long-term time evolution of the system. It can be proved rigorously that the equations of the model can predict the entire future of the system exactly and uniquely if we can specify the initial conditions exactly. In practice, however, we can never determine the initial conditions exactly in real systems.

The crucial difference between chaotic and ordinary dynamic evolution is the effect initial error has on predictability. In ordinary evolution, errors grow linearly and they can be corrected by subsequent observation. In chaotic evolution, errors grow exponentially rapidly and cannot be corrected. Chaotic systems are thus complex. This is so in spite of the fact that they can be specified by simple equations.

SAIFUL ISLAM

Research Unit Gottstein,

Max Planck Society,

Frankfurter Ring 243,

D 8000 Munich 40, FRG

\section{Before the event}

SIR-It seems that you are caught on the horns of a philosophical dilemma ("Down with the Big Bang", Nature 340, 425; 1989). You reject an oscillatory view of the Universe at least partly on the grounds that "the ultimate origin of our world cannot be discussed". You therefore reject as unsatisfactory an endless sequence of causes in a time which has no beginning. In the preceding paragraph, you object to the Big Bang because it implies the existence of an instant before which there was no time, and which is not susceptible to discussion of causes preceding the event. Surely this objection applies to any discussion that presumes to account for the "ultimate origin of our world"?

I have always thought it unlikely that a defence of the First Cause would ever appear in your columns. Now, however, I am not so sure.

MICHAEL WEBB

Molecular Biology Laboratory,

Sandoz Institute for Medical Research, 5 Gower Place, London WC1E 6BN, UK 